\title{
The ALPACA Project
}

\author{
Masato Takita ${ }^{a}$ for the ALPACA Collaboration \\ Institute for Cosmic Ray Research, the University of Tokyo, 5-1-5, Kashiwanoha, Kashiwa-shi, Chiba 277-8582, Japan
}

\begin{abstract}
We have started up the ALPACA (Andes Large area PArticle detector for Cosmic ray physics and Astronomy) project. The ALPACA experiment is composed of an $83,000 \mathrm{~m}^{2}$ air shower array and a $5,400 \mathrm{~m}^{2}$ underground muon detector array to make wide field-of-view high-sensitivity observations of highenergy cosmic rays/cosmic gamma rays on the Cerro Estuqueria highland, 4,740 $\mathrm{m}$ above sea level around Mount Chacaltaya, Bolivia. We briefly report on the design concept of the new project and its physics targets.
\end{abstract}

\section{Introduction}

In the southern hemisphere, the HESS group reported the discovery of 14 new gamma-ray sources by the galactic plane survey [1]. Surprisingly, most of them were UNIDentified (UNID) sources and faint in X-rays or other wavelengths. As the HESS survey was limited within the galactic plane in the southern hemisphere due to its narrow field of view, the importance of a wide field-ofview unbiased survey is emphasized. Furthermore, many of the 14 sources have a harder energy spectrum at $\mathrm{TeV}$ energies (indices: -1.8 to -2.8 ) than the standard Crab candle (index; -2.6). Although, the energy spectra were mostly measured below approximately $10 \mathrm{TeV}$ due to the sensitivity limit of the HESS air Cherenkov telescopes, they suggest that they extend up to the $100 \mathrm{TeV}$ region. The number of $\mathrm{TeV}$ sources now amounts to approximately 200. However, the origin of the cosmic rays has not yet been clarified.

Cosmic rays are supposed to be accelerated up to the knee energy (PeV) region at supernova remnants (SNRs) in our galaxy. Therefore, we naturally expect gamma rays at $100 \mathrm{TeV}$ energies, which originate in $\pi^{0}$ decays produced by the accelerated cosmic rays interacting with matter surrounding the SNRs. However, on-going experiments focus on measuring gamma rays in the $1-10 \mathrm{TeV}$ region. The gamma-ray emission of electron origin might be highly suppressed above $10 \mathrm{TeV}$ due to the rapid decrease of the inverse-Compton cross section by the Klein-Nishina effect as well as synchrotron radiation energy losses in the strong magnetic field around SNRs.

The detection and spectral measurement of gamma rays in the $100 \mathrm{TeV}$ region from their celestial sources, together with multi-wavelength (radio, X-ray, gamma-ray) observations, will be an important experiment enabling us to discriminate between the two processes (cosmicray/electron origins), to locate the acceleration site of cosmic rays (PeVatrons which accelerate cosmic rays up to $\mathrm{PeV}$ energies) and to verify the standard acceleration model. Some of the HESS 14 sources are very promising

a e-mail: takita@icrr.u-tokyo.ac.jp candidates for the cosmic-ray PeVatron. Furthermore, diffuse gamma rays from Fermi bubbles recently reported by the Fermi-LAT group and sub-PeV neutrino events [2] detected by IceCube suggests that Fermi bubbles are PeVatron candidates. Similarly, the energy spectrum of diffuse gamma rays from the extended region around the galactic center marginally measured by HESS up to approximately $10 \mathrm{TeV}$ also strongly indicates the existence of PeVatrons [3], from which we expect to detect gamma rays at $100 \mathrm{TeV}$ energies. Thus, a wide field-of-view gamma-ray imaging at $100 \mathrm{TeV}$ energies in the southern sky, where the HESS sources, the Fermi bubbles and the galactic center are located within the field of view, will be a key experiment.

\section{Experiment}

The experimental site (approximately $500 \mathrm{~m} \times 500 \mathrm{~m}$ $\sim 250,000 \mathrm{~m}^{2}$ in total area) is located on a flat high land called Cerro Estuqueria (4,740 $\mathrm{m}$ above sea level, $16^{\circ} 23^{\prime} \mathrm{S}$, $68^{\circ} 08^{\prime}$ W), as shown in Fig. 1, around Mount Chacaltaya, near La Paz, Bolivia. Our detectors will be set up in some part of this area.

We plan to set up a $5,400 \mathrm{~m}^{2}$ underground (approximately one to a few meters) muon detector array (MD) and an $83,000 \mathrm{~m}^{2}$ air shower array (AS), shown in Fig. 2. The MD of the water Cherenkov type is composed of eight pools with each pool (approximately $1 \mathrm{~m}$ deep) containing twelve $56 \mathrm{~m}^{2}$ unit detectors. AS is made up of $4011 \mathrm{~m}^{2}$ plastic scintillation counters at $15 \mathrm{~m}$ spacing.

The AS field of view is roughly 2 steradians. The expected angular resolution of AS is approximately 1 degree at $5 \mathrm{TeV}$ and 0.2 degrees around $100 \mathrm{TeV}$ for gamma rays. For $100 \mathrm{TeV}$ gamma rays, the AS energy resolution is estimated to be $\sim 25 \%$. The hadron rejection power of MD is more than $99.9 \%$ at $100 \mathrm{TeV}$, while keeping most of gamma-ray events. The long-term detector stability, angular resolution, pointing accuracy and energy scale can be calibrated by the cosmic ray shadow of the Moon as well as by some of the bright stable $\mathrm{TeV}$ gamma ray sources in the southern sky.

(C) The Authors, published by EDP Sciences. This is an Open Access article distributed under the terms of the Creative Commons Attribution License 4.0 (http://creativecommons.org/licenses/by/4.0/). 


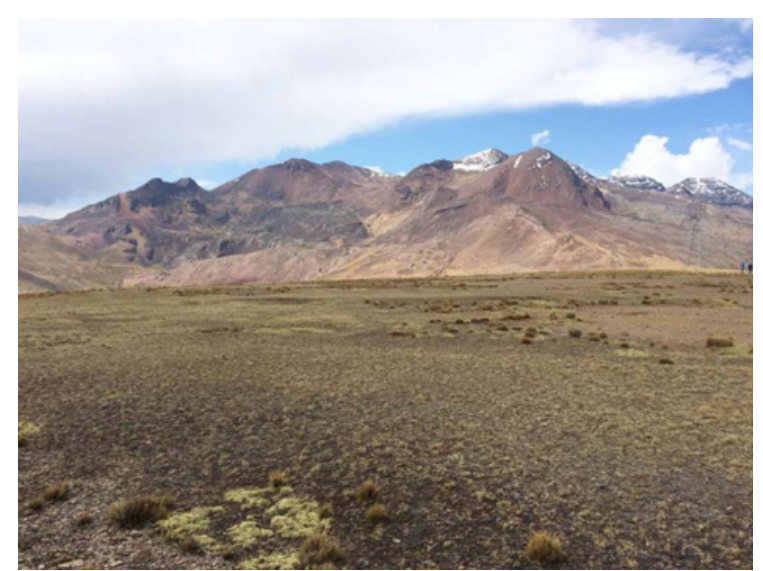

Figure 1. Experimental site for the ALPACA experiment, Cerro Estuqueria $\left(4,740 \mathrm{~m}\right.$ above see level, $\left.16^{\circ} 23^{\prime} \mathrm{S}, 68^{\circ} 08^{\prime} \mathrm{W}\right)$, near Mount Chacaltaya, in Bolivia.

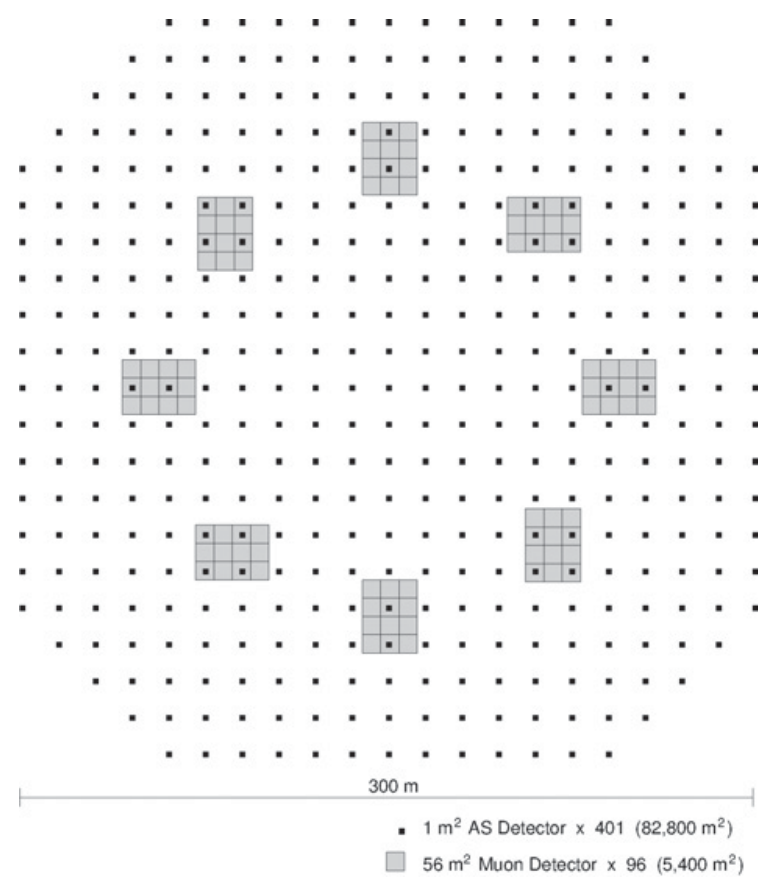

Figure 2. Schematic view of the ALPACA experiment. The small black squares indicate $4011 \mathrm{~m}^{2}$ plastic scintillator detectors, forming an air shower array with $83,000 \mathrm{~m}^{2}$ in area. The grey rectangles indicate eight underground muon detector pools, each of which contains twelve $56 \mathrm{~m}^{2}$ muon detector units. The total area of the underground muon detector array is $5,400 \mathrm{~m}^{2}$.

\section{Physics targets}

We have four physics targets in our new project:

1. Measurement of high-energy (5 TeV-1 PeV) cosmic gamma rays.

2. Measurement of cosmic ray energy spectra around the Knee energy region (100 TeV-100 PeV).

3. Measurement of cosmic ray anisotropy $>5 \mathrm{TeV}$ in the sidereal time frame.

4. Measurement of the Sun shadow in cosmic rays $>5 \mathrm{TeV}$.

We aim at low-background detection of celestial gamma rays in the $100 \mathrm{TeV}$ region with a world-best sensitivity

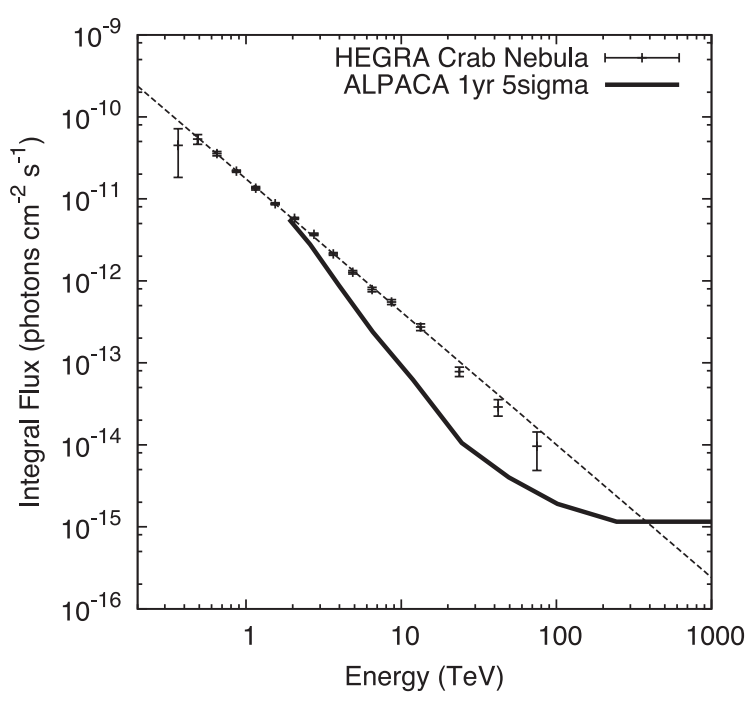

Figure 3. Sensitivity of ALPACA to high-energy gamma-ray point sources. The experimental points are from Ref. [4]. The ALPACA sensitivity is evaluated from Ref. [5].

(an order of magnitude better than any previous/existing experiments) and at locating the origins of cosmic rays accelerated up to the knee energy region in the southern sky. Around $100 \mathrm{TeV}$, the angular resolution of this air shower array is 0.2 degrees with a wide field-of-view of 2 steradians, while the energy resolution is estimated to be $25 \%$. The hadron rejection power of $\mathrm{MD}$ is more than $99.9 \%$ at $100 \mathrm{TeV}$, while keeping most of gamma-ray events. Assuming the presence of a Crab-like gamma-ray source in the southern sky, with a power-law differential energy spectrum proportional to $\mathrm{E}^{-2.6}$ extending up to above $100 \mathrm{TeV}$ where $\mathrm{E}$ is the energy of a gamma ray, the ALPACA experiment is sensitive to the source with $\sim 15 \%$ Crab intensity during one calendar year, as demonstrated in Fig. 3.

This southern-hemisphere AS + MD will be a unique experiment complementary to on-going experiments (FERMI, HESS, VERITAS, MAGIC, CALET, Tibet AS $\gamma$, HAWC) and future projects (LHAASO, CTA) in this field, which are either located in the northern hemisphere or aiming at gamma-ray astronomy below $10 \mathrm{TeV}$ region, or having a narrow field-of-view. Thus, the new energy window in the $100 \mathrm{TeV}$ region observing gamma rays with a wide field-of-view will be opened first in the southern sky by the ALPACA experiment. We expect to detect more than a dozen established sources, i.e., young SNRs (SN1006, RX J1713.7-3946, RX J0852.04622), Pulsar Wind Nebulae, the galactic center, etc in the $100 \mathrm{TeV}$ region, some of which may be cosmicray PeVatron candidates. Furthermore, our wide fieldof-view sensitivity to diffuse gamma rays allows us to study extremely diffuse gamma-ray sources which are difficult to detect by IACTs. The diffuse gamma rays from the Fermi bubbles recently reported by the FermiLAT group may be clearly detected, if they extend up to the $100 \mathrm{TeV}$ region. Similarly, the detection of diffuse gamma rays above $100 \mathrm{TeV}$ from an extended region from the galactic center is promising, where the gammaray energy spectrum strongly suggests the existence of PeVatrons. Detection and spectral measurement of gamma rays in the $100 \mathrm{TeV}$ region from these celestial sources, 
together with multi-wavelength observations (radio, X-ray, gamma-ray), are key points enabling us to discriminate between the two processes (cosmic-ray/electron origins), to locate the acceleration site of cosmic rays and to examine the standard acceleration model of cosmic rays.

From the astronomical point of view, we pioneer ultrahigh energy gamma-astronomy (above $100 \mathrm{TeV}$ ) in the southern sky. Besides, gamma-ray emission from nearby extragalactic sources, e.g., M87, Cen A, gamma rays of dark matter origin, those from the Sun disk recently obseved by Fermi [6] may be interesting subjects.

We also aim to measure the energy spectra of proton, helium and iron components separately around the knee energy region with the new AS + MD. The standard cosmic-ray acceleration model at SNRs predicts the knee energy of each nucleus component being proportional to $\mathrm{Z}$ (atomic number). We can discriminate proton and iron components by $\mathrm{MD}$, as an iron nucleus produces approximately twice as many muons as a proton with the same energy. Thus, the cosmic-ray acceleration scenario (SNR shock acceleration) will be verified by observing the linearly $\mathrm{Z}$ (atomic number)-dependent knee(= bent) positions of proton, helium, iron components around the knee energy region.

Precise cosmic-ray anisotropy measurements in the sidereal time frame in the $\mathrm{TeV}$ energy region in the southern sky provide unique data for the community to understand the magnetic field structure in the heliosphere. The ALPACA experiment gives complementary data in the $\mathrm{TeV}$ region to those from IceCube above a few tens of $\mathrm{TeV}$.

Furthermore, measurements of the Sun shadow in cosmic rays above the $\mathrm{TeV}$ energy region in the southern hemisphere also helps understand the modeling of the magnetic fields between the Sun and the Earth, complementary to the observations in the northern hemisphere.

\section{ALPAQUITA}

As a pilot experiment, the ALPAQUITA AS without MD, having $\sim 10 \%$ of the ALPACA AS in area will be constructed at the experimental site, Cerro Estuqueria, in 2017.

\section{Summary}

We have started up the ALPACA (Andes Large area PArticle detector for Cosmic ray physics and Astronomy) project which aims at imaging the southern sky by means of gamma rays in the $100 \mathrm{TeV}$ region, composed of a southern-hemisphere air shower array $\left(83,000 \mathrm{~m}^{2}\right)$ with a large $\left(5,400 \mathrm{~m}^{2}\right)$ underground water Cherenkov muon detector array in the Andes highland $(4,740 \mathrm{~m}$ above sea level) in Bolivia. The muon detector array enables us to discriminate the muon-poor gamma-ray component from the muon-rich hadronic component, allowing us to make low-background observations of gamma rays in the $100 \mathrm{TeV}$ region. This will be the first important attempt in the southern sky in this field. It should be stressed that, until now, there has not existed any high-sensitivity experiment capable of probing the gamma-ray southern sky around $100 \mathrm{TeV}$ with a wide field-of-view and high duty cycle.

The ALPACA experiment covers a wide range of cosmic ray physics, e.g., measurement of the chemical composition and energy spectra around the knee energy region, measurement of the cosmic ray anisotropy above the $\mathrm{TeV}$ energy region in the southern hemisphere, measurement of the Sun shadow in cosmic rays in the southern hemisphere, etc.

Thus, the expected impacts upon the field are promising, as the project gives an important clue to the problem which has remained over 100 years since the discovery of cosmic rays: the origin, acceleration and propagation mechanism of cosmic rays.

In 2017, we will construct the ALPAQUITA air shower array with $\sim$ one-tenth of the ALPACA air shower array in Cerro Estuqueria, as a pilot expriment.

\section{References}

[1] F. Aharonian et al., Science 307, 1938-1942 (2005)

[2] C. Lunardini et al., PRD 92, 021301-1-5 (2015)

[3] A. Abramoswski et al., Nature 531, 476 (2016)

[4] F. Aharonian et al., ApJ 614, 897 (2004)

[5] T.K. Sako et al., Astroparticle Physics 32, 177 (2009)

[6] C.Y. Kenny et al., arXiv: 1508.06276v1 